\title{
Epidemiology and Treatment of Distal Femoral Fractures in Adults: An Hospital Based Study
}

\author{
Rajendra Didel ${ }^{1}$, Kusum Choudhary ${ }^{2}$ \\ ${ }^{1}$ Assistant Professor, Department of Orthopedics, Government Medical College \& Bangur Hospital, Pali, Rajasthan, India, ${ }^{2}$ Resident, Department of Anaesthesia, S. \\ N. Medical College, Jodhpur, Rajasthan, India.
}

\section{Abstract}

Background: The frequency of these fractures is elevating with advancing age of the population, but they are less common than the proximal femur. After initial attempts of surgical management, high complication frequency were seen that severely affected the clinical outcomes. The present study was conducted with the aim to determine the epidemiology and the management strategies of distal femur fractures amongst subjects reporting to the hospital. Subjects and Methods: The present retrospective study analyzed 20 patients with distal femoral fractures managed by different types of surgical and non surgical methods during the period during a period of 4 years. The epidemiological variables studied were incidence, gender, age and circumstances. The type of management strategies employed were also noted. All the data thus obtained was arranged in a tabulated form and analyzed using SPSS software. Results: A total of 1050 subjects reported to the department with fractures, out of these there were 20 cases of distal femoral fractures. Upon classifying them according to Chiron's classification, 3 belonged to Chiron Group I1, 5 belonged to Chiron Group I2, 3 were of Chiron Group II 1 and majority were of Chiron group II 2. 16 were managed by osteosynthesis, out of which 13 were treated by internal plate blade $95^{\circ}, 2$ by condylar plate and 1 by external fixator. Conclusion: Fractures of distal femur need to dealt with great care in order to enable good quality of walk and knee function. In our study, majority of the subjects were managed by surgical protocols.

Keywords: Chiron, Distal, Osteosynthesis, Surgical.

Corresponding Author: Dr. Kusum Choudhary, Resident, Department of Anaesthesia, S. N. Medical College, Jodhpur, Rajasthan, India.

Received: March 2019

Accepted: March 2019

\section{Introduction}

Distal femoral fractures are not commonly seen but still are severe. ${ }^{[1]}$ Their incidence varies in different populations that were studied. In England, they represented 3-6\% of femoral fractures amongst adults and $0.4 \%$ of all the fractures studied and they represented $10 \%$ of all the femoral fractures. ${ }^{[1-4]}$ The frequency of these fractures is elevating with advancing age of the population, but they are less common than the proximal femur. In most of the cases, they are because of high-energy accidents and their management is generally surgical. Distal femoral fractures occur in a bimodal distribution; commonly between 15 and 50 years of age, chiefly afflicting male subjects, due to highenergy impacts, and amongst subjects above 50 years of age, mostly affecting female subjects with osteoporosis, due to comparatively low-energy trauma. ${ }^{[5-7]}$ Majority of the classification systems categorize distal femur fractures into three prime types: unicondylar, extraarticular, and bicondylar. ${ }^{[8]}$ Back in the 1970s, fractures of distal femur were managed with open reduction and fixation with different established methods and implants. After initial attempts of surgical management, high complication frequency were seen that severely affected the clinical outcomes. The present study was conducted with the aim to determine the epidemiology and the management strategies of distal femur fractures amongst subjects reporting to the hospital.

\section{Subjects and Methods}

The present retrospective study analyzed 20 patients with distal femoral fractures managed by different types of surgical and non surgical methods during the period during a period of 4 years. Enrolled subjects hospitalized with distal femoral fractures with a follow up duration of more than 12 months. The subjects whose pre or post operative radiographic records were missing were not included in the study. The subjects who did not meet the follow up criteria were excluded from the study. Classification by Chiron was used to categorize the patients. The surgical approach for the management which included fixation with metallic implants in case of closed fractures and external fixation in case of open fractures and in trans calcaneal traction in orthopedic management protocol. The rehabilitation of knee -both active and passive was initiated early after the surgical management and was continued in the physiotherapy department. The epidemiological variables studied were incidence, gender, age and circumstances. The type of 
management strategies employed were also noted. All the data thus obtained was arranged in a tabulated form and analyzed using SPSS software.

\section{Results}

A total of 1050 subjects reported to the department with fractures, out of these there were 20 cases of distal femoral fractures. There were 12 males and 8 females amongst them. The age range of the subjects were 19-72 years with the mean age of 38.2 years. Right side was fractured amongst 11 patients and left side was injured amongst 9 patients. Upon classifying them according to Chiron's classification, 3 belonged to Chiron Group I1, 5 belonged to Chiron Group I2, 3 were of Chiron Group II 1 and majority were of Chiron group II 2. [Table 1]

[Table 2] illustrates the management strategy followed amongst the subjects. 16 were managed by osteosynthesis, out of which 13 were treated by internal plate blade $95^{\circ}$, 2 by condylar plate and 1 by external fixator. There was 1 case of whose treatment was orthopedic. The mean time of fracture union was 20 days.

\begin{tabular}{l}
\hline Table 1: demographics and type of distal femoral fractures \\
\begin{tabular}{|l|l|}
\hline Variable & Frequency \\
\hline Gender & 12 \\
\hline Male & 8 \\
\hline female & $19-72$ years \\
\hline Age range & 11 \\
\hline Side & 9 \\
\hline Right & \multicolumn{1}{|l}{} \\
\hline left & 3 \\
\hline Classification & 5 \\
\hline Chiron group I 1 & 3 \\
\hline Chiron group I 2 & 8 \\
\hline Chiron group II 1 & 1 \\
\hline Chiron group II 2 & \\
\hline Chiron group II 5 &
\end{tabular}
\end{tabular}

Table 2: Type of management performed

\begin{tabular}{|c|c|}
\hline Treatment protocol & Frequency \\
\hline Osteosynthesis & 16 \\
\hline internal plate blade $95^{\circ}$ & 13 \\
\hline condylar plate & 2 \\
\hline external fixator & 1 \\
\hline Orthopedic & 1 \\
\hline
\end{tabular}

\section{Discussion}

Distal femoral fractures are intricate injuries that are tuff to be managed and may lead to long-term disability and elevated morbidity. They contribute to $4-7 \%$ of all femur fractures and account for $31 \%$ of the femur fractures after excluding hip fractures [9-13]. Before 1970, majority of distal femoral fractures were managed with conservative approaches like skeletal traction and bracing till the time of healing of fracture that produced favorable results, that was at the expense of extensive hospitalization and bargained knee motion. ${ }^{[8,9]}$ After that, alternative methodology for unstable distal femoral fractures were identified, that included double plating, usage of endosteal substitutions, and anatomically shaped plates. ${ }^{[14,15]}$ In 1980s, various advances in fracture management were used to manage these difficult injuries and the clinical outcomes were improvised. Indirect reduction and improvised maintenance of biology of fracture biology was given by Mast et al. ${ }^{[16]}$ Various different methods of fixation methods have been elaborated for the treatment of fractures of distal femur like $95^{\circ}$ angled blade plate, condylar buttress plate, dynamic condylar screw, , and retrograde nails. ${ }^{[15-17]}$ The lock plating systems have been found in Davos Switzerland in the 1990s, that screws the lock to the plate leading to formation of a multiple fixed-angle contact that avoids compression of the periosteum, thus maintaining the vascularity of the injured bone. ${ }^{[18,19]}$ The incidence of distal femoral fractures was slightly lower in our study compared to the data in the literature with figures varying between $3 \%$ to $10 \%{ }^{[1-3]}$ The incidence elevates with the advanced aging of the populace in the European nations, in association with the increased rate of osteoporosis, but lesser than those of proximal femoral fractures. ${ }^{[20]}$ The average age in the present study was 38.2 years, similar to results by Bedes L. and al. ${ }^{[21]}$ that reported 39.6 years and Sié Essoh J.B. ${ }^{[22]}$ that showed average age of 44 years. On the contrary, Pascarella and al. ${ }^{[23]}$ Rasmus Elsoe et al. ${ }^{[24]}$ showed average age of 62 years and 62.2 years in their studies respectively. As per Pietu and al average age average age of 63.5 years was observed for men and 75 years for females. The difference was seen amongst developed and developing nations. Road traffic accidents were the main cause of fractures in the present study. It was similar to study by Sié Essoh J.B et al. ${ }^{[22]}$ On the contrary, Chantelot C. and al. ${ }^{[25]}$ reported a predominance of falls amongst patients over 80 years old.

\section{Conclusion}

Fractures of distal femur need to dealt with great care in order to enable good quality of walk and knee function. In our study, majority of the subjects were managed by surgical protocols. Better and up to date materials should be used for optimum treatment of the subjects.

\section{References}

1. Ehlinger M, Ducrot G, Adam P, Bonnemet F. Distal femur fractures. Surgical techniques and a review of the literature. Orthopaedics and Traumatology: Surgery and Research. 2013; 99:353-360.

2. Lauper N, Sava D, Hoffmeyer P. Fractures of the knee area in the elderly: management and evolution. Rev Med Switzerland. 2012; 8:2434-2437

3. Ascencio G, Bertin R, Mergy B. Fractures of the lower extremity of the femur. Encyclical. Med Chir App Locomotive. 1995; 14:80-A-10.

4. Kolmert L, Wulff K. Epidemiology and treatment of distal femoral fractures in adults. Acta Orthop Scand. 1982; 53(6):957962.

5. Giles JB, DeLee JC, Heckman JD, Keever JE. Supracondylar- 
intercondylar fractures of the femur treated with a supracondylar plate and lag screw. J Bone Joint Surg Am 1982; 64:864-870.

6. Kammerlander C, Riedmüller P, Gosch M, Zegg M, Kammerlander-Knauer U, Schmid R, et al. Functional outcome an mortality in geriatric distal femoral fractures. Injury 2012; 43:1096-1101.

7. Wähnert D, Hoffmeier K, Fröber R, Hofmann GO, Mückley T. Distal femur fractures of the elderly - Different treatment options in a biomechanical comparison. Injury 2011; 42:655-659.

8. Butt MS, Krikler SJ, Ali MS. Displaced fractures of the distal femur in elderly patients: operative versus non-operative treatment. J Bone Joint Surg Br 1996; 78:110-114.

9. Schatzker J, Lambert DC. Supracondylar fractures of the femur. Clin Orthop Relat Res 1979; 138:77-83.

10. Arneson TJ, Melton LJ 3 rd, Lewallen DG, O'Fallon WM. Epidemiology of diaphyseal and distal femoral fractures in Rochester, Minnesota 1965-1984. Clin Orthop Relat Res 1988; 234:188-194.

11. Giles JB, DeLee JC, Heckman JD, Keever JE. Supracondylarintercondylar fractures of the femur treated with a supracondylar plate and lag screw. J Bone Joint Surg Am 1982; 64:864-870.

12. Heiney JP, Battula S, O'connor JA, Ebraheim N, Schoenfeld AJ, Vrabec G. Distal femoral fixation: a biomechanical comparison of retrograde nail,retrograde intramedullary nail, and prototype locking retrograde nail. Clin Biomech 2012; 27:692-696.

13. Kolmert L, Wulff K. Epidemiology and treatment of distal femoral fractures in adults. Acta Orthop Scand 1982; 53:957-962.

14. Siliski J, Mahring M, Hofer HP. Supracondylar-intercondylar fractures of the femur. Treatment by internal fixation. J Bone Joint Surg Am 1989; 71:95-104.

15. Sanders R, Swiontkowski M, Rosen H, Helfet D. Double-plating of comminuted, unstable fractures of the distal part of the femur. J Bone Joint Surg Am 1991; 73:341-346.

16. Mast J Jakob R, Ganz R. Planning and reduction technique in fracture surgery. Berlin: Springer-Verlag; 1989. 140-187.

17. Mallina R, Kanakaris NK, Giannoudis PV. Peri-articular fractures of the knee: an update on current issues. Knee 2010; 17:181-186.

18. Christodoulou A, Terzidis I, Ploumis A, Metsovitis S, Koukoulidis A, Toptsis C. Supracondylar femoral fractures in elderly patients treated with the dynamic condylar screw and the retrograde intramedullary nail: a comparative study of the two methods. Arch Orthop Trauma Surg 2005; 125:73-79.

19. Ali I. Surgical outcome of supracondylar and intercondylar fractures femur in adults treated with dynamic condylar screw. JPMI 2011; 25:49-55.

20. Pietu G, Lebaron M, Flecher X, Hulet C, Vandenbussche E. Sofcot. Epidemiology of distal femur fracturs in France in 2011-12. Orthopaedics and traumatology: Surgery and research. 2014; 100:545-548

21. Bedes L, Bonnevialle P, Ehlinger M. External fixation of distal femoral fractures in adults multicentre retrospective study of 43 patients. Orthopaedics and traumatology: Surgery and research. 2013; 100:867-872.

22. Sié Essoh JB, Mobiot CA, Traoré A, Lambin Y. Distal femoral fractures treated with condylar buttres plate in a West African hospital. J clin Orthop Trauma. 2012; 3(2):98-102.

23. Pascarella R, Bettuzzi C, Bosco G. Results in treatment of distal fractures using polyaxial locking plate. Strategies. Trauma Limb Reconstr. 2014; 9(1):13-18.

24. Elsoe R, Ceccottip AA, Larsen P. Population - based epidemiology and incidence of distal femur fractures. International Orthopaedics. 2018; 42(1):191-196.

25. Chantelot C, Dumont G, Ehlinger M. Fractures of the distal femur beyond 80 years: about a multicenter study of a prospective series. Geriatrics Review. 2015; 40(6):327-333.

Copyright: (C) the author(s), 2019. It is an open-access article distributed under the terms of the Creative Commons Attribution License (CC BY 4.0), which permits authors to retain ownership of the copyright for their content, and allow anyone to download, reuse, reprint, modify, distribute and/or copy the content as long as the original authors and source are cited.

How to cite this article: Didel R, Choudhary K. Epidemiology and Treatment of Distal Femoral Fractures in Adults: An Hospital Based Study. Asian J. Med. Res. 2019;8(1):OR12-OR14.

DOI: dx.doi.org/10.21276/ajmr.2019.8.1.OR5 